\begin{tabular}{|c|c|}
\hline Title & Linear temperature term of heat capacity in insulating and superconducting $\mathrm{La} B a \mathrm{Cu}-\mathrm{O}$ systems \\
\hline Author(s) & Kumagai, K.; Nakamichi, Y.; W atanabe, I.; Nakamura, Y .; Nakajima, H.; W ada, N.; Lederer, P. \\
\hline Citation & $\begin{array}{l}\text { PHY SICA L REVIEW LETTERS, 60(8), } 724.727 \\
\text { https://doi.org/10.1103/PhysRevLett.60.724 }\end{array}$ \\
\hline Issue Date & $1988-02$ \\
\hline Doc URL & http:/hdl .handle.net/2115/5894 \\
\hline Rights & Copyright $\odot 1988$ A merican Physical Society \\
\hline Type & article \\
\hline File Information & PRL60-8.pdf \\
\hline
\end{tabular}

Instructions for use 


\title{
Linear Temperature Term of Heat Capacity in Insulating and Superconducting La-Ba-Cu-O Systems
}

\author{
K. Kumagai, Y. Nakamichi, I. Watanabe, Y. Nakamura, H. Nakajima, and N. Wada \\ Department of Physics, Faculty of Science, Hokkaido University, Sapporo 060, Japan
}

and

\author{
P. Lederer ${ }^{(\mathrm{a})}$
}

Institute for Solid State Physics, The University of Tokyo, Roppongi, Minato-ku, Tokyo 106, Japan

(Received 13 January 1988)

\begin{abstract}
The heat capacity of $\mathrm{La}_{2}-\mathrm{Ba}_{x} \mathrm{CuO}_{4}$ was investigated between 0.8 and $10 \mathrm{~K}$. The linear temperature term $\gamma T$ of the heat capacity is absent in the antiferromagnetic-ordered region of $x \leqq 0.02 . \gamma$ increases abruptly from $x=0.02$ to $x=0.04$ where the system is still insulating at low temperature, and seems to saturate at the order of $5 \mathrm{~mJ} / \mathrm{mol} \cdot \mathrm{K}^{2}$ in the superconducting region of $x \geqq 0.05$. The correlation between magnetic order and vanishing $\gamma T$ term and the existence of a finite $\gamma T$ term for $\mathrm{Ba}$ doping give support to the resonating-valence-bond picture.
\end{abstract}

74.70.Hk, 74.30.Ek, 75.50.Ee

The discovery of high- $T_{c}$ superconductors ${ }^{1,2}$ has generated much interest in the basic properties of superconducting oxides and possible new mechanisms for high- $T_{c}$ superconductivity. ${ }^{3}$ In the $\mathrm{La}_{2} \mathrm{CuO}_{4}$ system, the antiferromagnetic (AF) ordering has been confirmed by measurements of magnetic susceptibility, ${ }^{4}$ neutron scattering, ${ }^{5}$ muon spin relaxation, ${ }^{6}$ and nuclear quadrupole resonance (NQR). ${ }^{7,8}$ The magnetism is highly two dimensional in character and is expected to play a key role in the understanding of high- $T_{c}$ superconductivity in the La-Ba-Cu-O and Y-Ba-Cu-O systems. ${ }^{9}$ The recent study of neutron scattering of $\mathrm{La}_{2} \mathrm{CuO}_{4}$ has revealed novel two-dimensional antiferromagnetic behavior. ${ }^{10}$ Anomalies of the nuclear relaxation rates in $\mathrm{Ba}$-doped $\mathrm{La}_{2} \mathrm{CuO}_{4}$ have been found in the region between antiferromagnetic and superconducting states. ${ }^{11}$

Anderson ${ }^{3}$ proposed that the undoped $\mathrm{La}_{2} \mathrm{CuO}_{4}$ system has a resonating valence-bond (RVB) ground state, and that high- $T_{c}$ superconductivity develops as a result of doping of this new type of insulator. A striking consequence of the theory is that spin and charge degrees of freedom are decoupled in the RVB state. Although a large gap exists for charge excitations in the undoped materials, spin excitations behave like a gas of neutral massless fermions ${ }^{3,12,13}$ with a bandwidth of order $J$, the exchange energy of the problem. Therefore, down to the lowest temperature, a heat-capacity term linear in temperature, $C=\gamma T$, should be observed with $\gamma \sim J^{-1}$ on the order of $\frac{1}{2000} \mathrm{~K}$.

In the case of doping, mean-field theory ${ }^{14-17}$ leads to a gap for spinons (the neutral spin excitations of the theory), but Anderson ${ }^{18}$ has argued that, because of the SU(2) symmetry of the spinons, the gaplessness of the spinon excitation spectrum persists within the superconducting and within the "normal" RVB phases, so that the $\gamma T$ term should be observed whenever the resonating singlet liquid is the doped or undoped ground state. The fact that antiferromagnetism has been proved to exist experimentally ${ }^{4-8}$ in undoped and slightly doped $\mathrm{La}_{2-x}$ $M_{x} \mathrm{CuO}_{4}$ (with $M=\mathrm{Ba}$ or $\mathrm{Sr}$ ) does not ruin the $\mathrm{RVB}$ picture, since the materials are $3 \mathrm{D}$ anisotropic layered materials. A small coupling $J_{\perp} \ll J$ between layers may counterbalance the energy difference between RVB and AF within each layer.

The sensitivity of the observed antiferromagnetic order to doping ${ }^{4,7,8}$ can be understood if RVB is more stable than $\mathrm{AF}$ in $2 \mathrm{D}$, in which case our decreasing the exchange coupling of the $3 \mathrm{D}-\mathrm{AF}$ state by decreasing the average spin per site may result in the stabilization of RVB again at small doping. Alternatively, one may speculate about the destruction of spinon density-wave ordering by changes of the pseudo-Fermi-surface nesting due to doping. ${ }^{9}$ Whatever the mechanism which allows a small hole concentration to destabilize AF, RVB predicts no $\gamma T$ term in the heat capacity of the AF phase. Such terms can only appear if magnetic ordering is destroyed, provided a pseudo-Fermi surface continues to exist in the doped materials.

$\gamma T$ terms have been found with values ranging from 0.5 to $1.8 \mathrm{~mJ} / \mathrm{mol} \cdot \mathrm{K}^{2}$ in $\mathrm{La}_{2} \mathrm{CuO}_{4-y}{ }^{19}$ to values between 10 and $20 \mathrm{~mJ} / \mathrm{mol} \cdot \mathrm{K}^{2}$ in $\mathrm{YBa}_{2} \mathrm{Cu}_{3} \mathrm{O}_{7-y}$, whether superconducting ${ }^{20}$ or insulating. ${ }^{21}$ Values of $5 \mathrm{~mJ} / \mathrm{mol} \cdot \mathrm{K}^{2}$ are reported for $\mathrm{La}_{2-x} M_{x} \mathrm{O}_{4}$ with $x=0.15$ and $M=\mathrm{Sr}$ or $\mathrm{Ba}^{22}$ The poor reproducibility of $\gamma$ values has led many to believe that the $T$-linear terms were of extrinsic origin, and could be due to various inhomogeneities such as a high density of two-level systems (TLS) ${ }^{23}$ which also lead to $\gamma T$ terms, of the order of $1 \mathrm{~mJ} / \mathrm{mol} \cdot \mathrm{K}^{2}$, in the heat capacity of glasses, insulating or metallic disordered solids, and some ceramics. ${ }^{24}$

In one case of simultaneous measurements of magnetic susceptibility and heat capacity on the same sample, ${ }^{4,25}$ a correlation seemed to exist between magnetic ordering and small $\gamma\left(<1 \mathrm{~mJ} / \mathrm{mol} \cdot \mathrm{K}^{2}\right)$ on one hand, and super- 


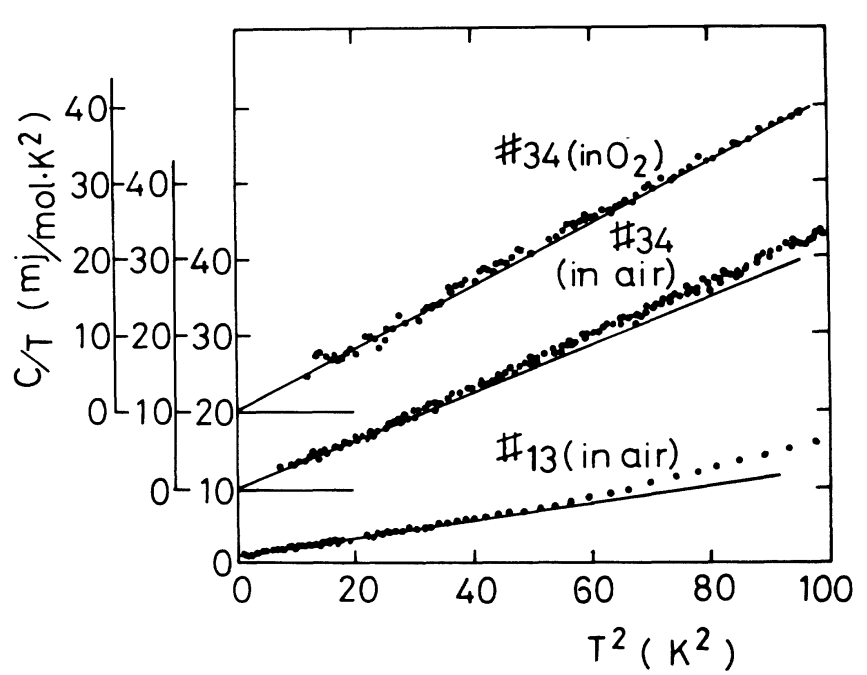

FIG. 1. Heat capacity plotted as $C / T$ vs $T^{2}$ for three undoped $\mathrm{La}_{2} \mathrm{CuO}_{4}$ samples.

conducting state and large $\gamma\left(5 \mathrm{~mJ} / \mathrm{mol} \cdot \mathrm{K}^{2}\right)$, on the other hand. This seems to us to suggest that such terms are intrinsic, since magnetic ordering, and magnetic ordering alone, is capable of suppressing them, as mentioned above. Thus, we were prompted to study the possible correlation between $\gamma$ and antiferromagnetism in $\mathrm{La}_{2-x} \mathrm{Ba}_{x} \mathrm{CuO}_{4}$.

Samples were prepared by our reacting the mixture of $\mathrm{La}_{2} \mathrm{O}_{3}, \mathrm{BaCO}_{3}$, and $\mathrm{CuO}$ at $900^{\circ} \mathrm{C}$ in air for $12 \mathrm{~h}$, sintering in pellet form at $1100^{\circ} \mathrm{C}$, and annealing the pellet at $600^{\circ} \mathrm{C}$ for $12 \mathrm{~h}$. Heat treatment for most samples was done in air atmosphere. A part of one sample $\left(\mathrm{La}_{2} \mathrm{CuO}_{4}\right.$ No. 34) was annealed at $600^{\circ} \mathrm{C}$ for $200 \mathrm{~h}$ in oxygen atmosphere. The electrical resistance was measured by the standard four-wire method with ac and dc current of 0.1-10 mA. The magnetic susceptibility was also preliminarily measured. We confirmed the bulk superconductivity for $x \geqq 0.05$. Heat-capacity measurements were made with the two different cryostats with a heat-pulse method. A ${ }^{3} \mathrm{He}$ cryostat with a calibrated carbon thermometer was used to measure down to $0.8 \mathrm{~K}$. The heat capacity was also measured in another cryostat with a $\mathrm{Ge}$ thermometer down to $2 \mathrm{~K}$ and the results were compared with each other.

Figure 1 show the heat capacities of three undoped $\mathrm{La}_{2} \mathrm{CuO}_{4}$ samples by plots of $C / T$ vs $T^{2}$. The heat capacities of $\mathrm{La}_{2} \mathrm{CuO}_{4}$ are somewhat different from sample to sample, and seem to depend on the sample quality. The temperature dependence of heat capacity is fitted by the relation $C=\gamma T+\beta T^{3}$ by the method of least squares in the low-temperature region. The heat capacity of No. 34 in $\mathrm{O}_{2}$ atmosphere is well fitted from the highest temperature of $10 \mathrm{~K}$. Here the $T^{3}$ term is from the phonon contributions in the Debye model. From the coefficients of the $T^{3}$ term in the low-temperature region, we deduce values of the Debye temperature of 240,190 , and $163 \mathrm{~K}$

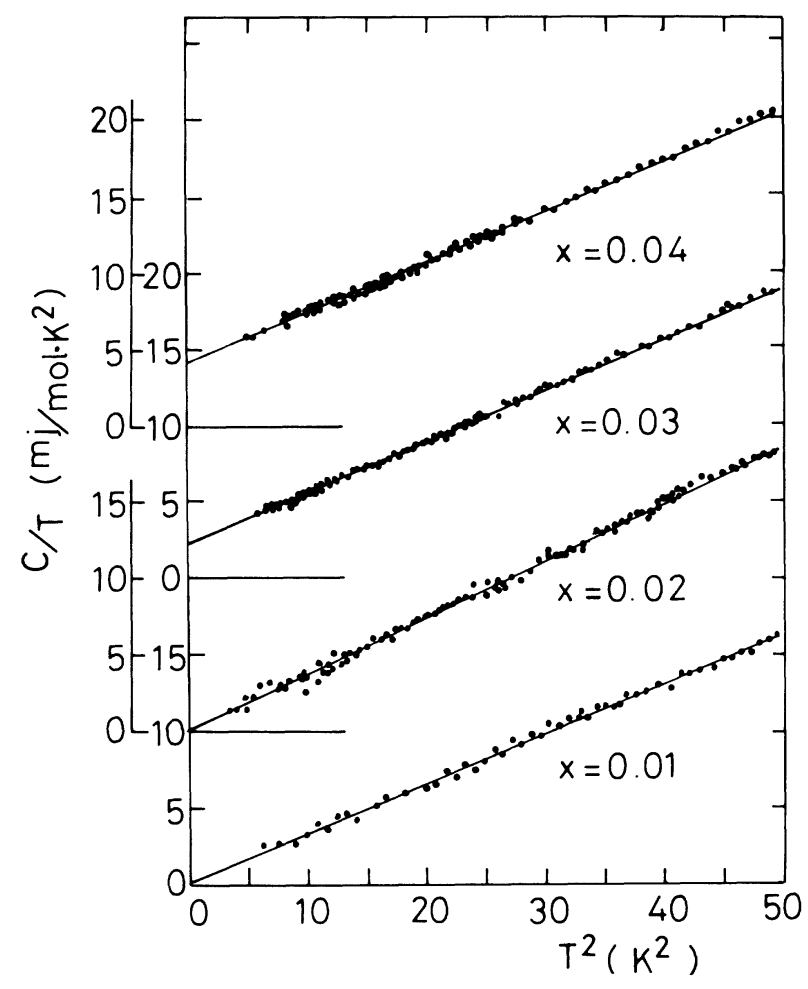

FIG. 2. Heat capacity plotted as $C / T$ vs $T^{2}$ for nonsuperconducting $\mathrm{La}_{2}-\mathrm{Ba}_{x} \mathrm{CuO}_{4}$. The solid lines are obtained by least-squares fitting below $7 \mathrm{~K}$.

for samples No. 13, No. 34 (annealed in air), and No. 34 (annealed in oxygen), respectively. The magnitude of the $T$-linear term, $\gamma$, is obtained from extrapolation to $T=0$ by least-squares fitting. The $\gamma$ values are at most $0.4 \mathrm{~mJ} / \mathrm{mol} \cdot \mathrm{K}^{2}$ for sample No. 13 and between 0.0 and $0.5 \mathrm{~mJ} / \mathrm{mol} \cdot \mathrm{K}^{2}$ for sample No. 34. Thus, $\gamma$ in $\mathrm{La}_{2} \mathrm{CuO}_{4}$ is less than $0.5 \mathrm{~mJ} / \mathrm{mol} \cdot \mathrm{K}^{2}$ within our experimental uncertainty.

Figure 2 shows the results of heat capacity of the $\mathrm{Ba}$ doped samples by plots of $C / T$ vs $T^{2}$. The samples shown in this figure are still insulating as demonstrated by the measurements of electrical resistance. ${ }^{26}$ The heat capacities are fitted by the relation $C=\gamma T+\beta T^{3}$ below $7 \mathrm{~K}$. The Debye temperature is almost $180 \mathrm{~K}$, independent of $\mathrm{Ba}$ concentration. $\gamma$ is between 0 and $0.5 \mathrm{~mJ} /$ $\mathrm{mol} \cdot \mathrm{K}^{2}$ for $X<0.02$ and increases rapidly up to $4-5$ $\mathrm{mJ} / \mathrm{mol} \cdot \mathrm{K}^{2}$ with increasing $x$. Figure 3 shows the $C / T$ vs $T^{2}$ plots for superconducting samples. The heat capacity in the superconducting state is similar to that reported previously. ${ }^{22,25} \gamma$ for $x=0.075$ is rather small compared to that for the other superconducting samples, but is still $2 \mathrm{~mJ} / \mathrm{mol} \cdot \mathrm{K}^{2}$. The values of $\gamma$ are shown in Fig. 4 as a function of $\mathrm{Ba}$ concentration together with the superconducting transition temperatures obtained by the electrical resistance measurement. ${ }^{8,11}$ The boundary of the antiferromagnetic phase in Fig. 4 is obtained by the NQR results ${ }^{8,11}$ for the same samples as the ones in the 


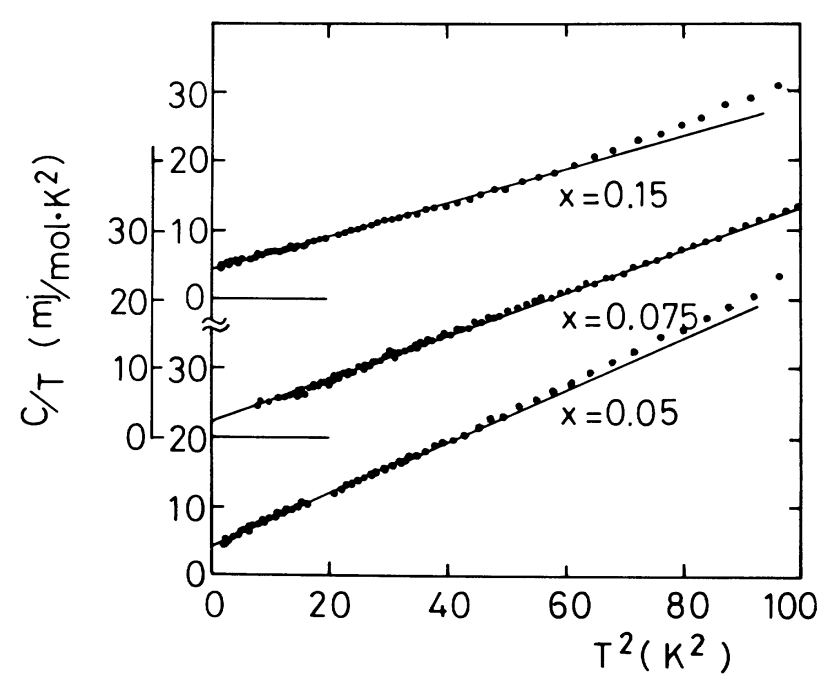

FIG. 3. Heat capacity plotted as $C / T$ vs $T^{2}$ for superconducting $\mathrm{La}_{2}{ }_{x} \mathrm{Ba}_{x} \mathrm{CuO}_{4}$. The solid lines are obtained by leastsquares fitting in the lowest-temperature region.

present study.

The samples prepared by reacting in air atmosphere have few oxygen vacancies and many grain boundaries. Oxygen deficiencies are considered to increase with $\mathrm{Ba}$ doping for $x>0.15$ but do not change much for small $x{ }^{27}$ Supposing such a defect structure could give rise to a tunneling $T$-linear term, $\gamma$ would be expected to change linearly or smoothly with small doping of Ba. The large sample dependence of $\gamma$ would also be expected. In the present samples of $\mathrm{La}_{2} \mathrm{CuO}_{4}$ with different preparation, the values of $\gamma$ are scattered within at most $0.5 \mathrm{~mJ} / \mathrm{mol} \cdot \mathrm{K}^{2}$ which is small compared to the order of $5 \mathrm{~mJ} / \mathrm{mol} \cdot \mathrm{K}^{2}$ for $x>0.02$. We see no reason for the contribution to $\gamma$ due to TLS to increase abruptly from the concentration of $x=0.02$. Therefore, it seems that the contribution to the heat capacity due to the TLS origin is not sizable for the $\mathrm{La}-\mathrm{Ba}-\mathrm{Cu}-\mathrm{O}$ system and that the relatively large $T$-linear term for $x>0.02$ arises from another origin.

It should be noted that $\gamma$ is small or zero in the antiferromagnetic region and increases when the antiferromagnetic order disappears. Thus, our data seem to indicate a fairly strong correlation between magnetic ordering and vanishingly small (within experimental accuracy) $\gamma T$ term. This suggests an intrinsic origin for the $\gamma T$ term, when it is finite, and gives support to the RVB picture. The value for $J$ which corresponds to $\gamma=5$ $\mathrm{mJ} / \mathrm{mol} \cdot \mathrm{K}^{2}$ is $\simeq 1000 \mathrm{~K}$.

In the concentration range $0.01 \leqq x \leqq 0.05$, we have confirmed the anomalies of nuclear relaxation rates which are strongly peaked at a temperature $T_{c}^{*} .{ }^{11}$ This range is an intermediate one between vanishing $\gamma$ in AF $(x \leqq 0.02)$ and large $\gamma$ in the superconducting state $(x \geqq 0.05)$. It seems that relaxation anomalies, clearly

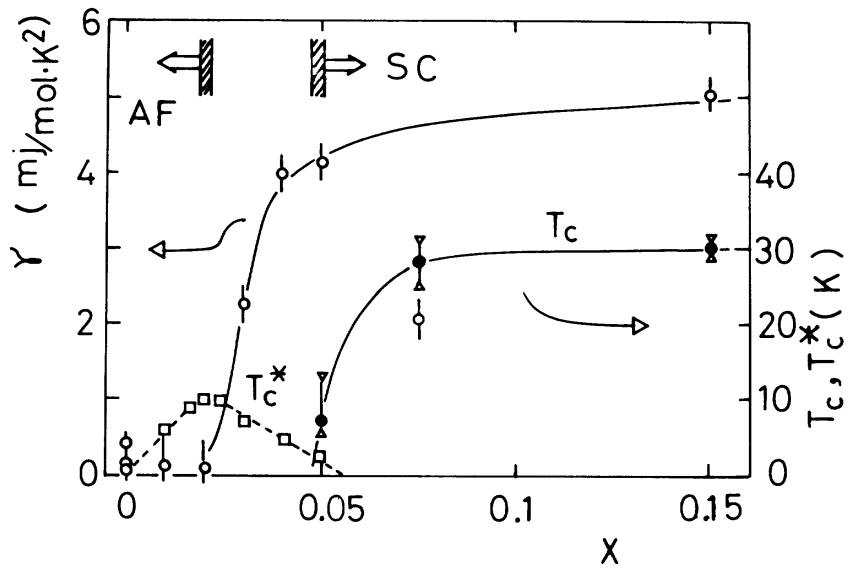

FIG. 4. Concentration dependence of the coefficients of the $T$-linear term of heat capacity, $\gamma$, of $\mathrm{La}_{2-x} \mathrm{Ba}_{x} \mathrm{CuO}_{4}$. Superconducting transition temperatures, $T_{c}$, are also shown. Inverted triangles are from the onset of resistivity, filled circles are from midpoint, and triangles are from $10 \%$ above zero resistivity. The boundary of the antiferromagnetic phase is from the NQR results (Ref. 8). $T_{c}^{*}$ (squares) is the temperature at which nuclear relaxation rates show the anomalies (Ref. 11).

associated with the fluctuations of spins, correspond to partial suppression of spinon density of states. Interestingly, such anomalous behavior of spin degrees of freedom at low temperature is not associated with anomalies in the heat capacity at $T_{c}^{*}$.

Evidence against the above interpretation of our data is the observation of antiferromagnetic ordering in the insulating compound $\mathrm{YBa}_{2} \mathrm{Cu}_{3} \mathrm{O}_{6}{ }^{28}$ A large $\gamma$ term (15 $\mathrm{mJ} / \mathrm{mol} \cdot \mathrm{K}^{2}$ ) has been observed in a compound with the same composition. ${ }^{21}$ This crucial point deserves careful experimental investigation. There is no evidence that the sample ${ }^{21}$ with large $\gamma$ was antiferromagnetically ordered, and no evidence that the antiferromagnetically ordered sample $^{28}$ had a large $\gamma$ term. Given the metallurgical uncertainties and the possible occurrence of large metastability of competing RVB and AF ground states, it is necessary to measure heat capacity and possible antiferromagnetism on the same sample. Only then shall we know if the properties of $\mathrm{YBa}_{2} \mathrm{Cu}_{3} \mathrm{O}_{6}$ ruin the correlation which we have found between antiferromagnetism and vanishing $\gamma$ in the insulating oxides. It is also necessary to check that different sample preparation procedures do not change the results qualitatively.

The extensive investigation of heat capacity associated with the NQR anomalies ${ }^{11}$ will be given separately.

The authors would like to thank Professor H. Fukuyama, Professor T. Fujita, and Professor Y. Maeno for helpful discussions and comments. This work was supported in part by a special Grant-in Aid for promotion of education and science in Hokkaido University provided by the Ministry of Education, Science and Culture of Japan. One of authors (P.L.) thanks the University of Tokyo for financial support. 
(a) On leave of absence from Physique des Solides, Bât. 510, Université Paris Sud, 91405 Orsay, France.

${ }^{1}$ J. G. Bednorz and K. A. Müller, Z. Phys. B 64, 198 (1986).

${ }^{2}$ C. W. Chu, P. H. Hor, R. L. Meng, Z. J. Huang, and Y. Q.

Wang, Phys. Rev. Lett. 58, 405 (1987).

${ }^{3} \mathrm{P}$. W. Anderson, Science 235, 1196 (1987).

${ }^{4}$ T. Fujita et al., Jpn. J. Appl. Phys. 26, L368 (1987).

${ }^{5}$ D. Vaknin et al., Phys. Rev. B 58, 2802 (1987).

${ }^{6}$ Y. Uemura et al., Phys. Rev. Lett. 59, 1045 (1987).

${ }^{7}$ Y. Kitaoka et al., J. Phys. Soc. Jpn. 46, 3024 (1987).

${ }^{8}$ I. Watanabe et al., J. Phys. Soc. Jpn. 46, 3028 (1987).

${ }^{9}$ Y. Hasegawa and Y. Fukuyama, Jpn. J. Appl. Phys. 26, 1099 (1987).

${ }^{10}$ G. Shirane, Y. Endoh, R. J. Birgeneau, M. A. Kastner, Y. Hidaka, M. Oda, M. Susuki, and T. Murakami, Phys. Rev. Lett. 59, 1613 (1987).

${ }^{11}$ K. Kumagai, I. Watanabe, H. Aoki, Y. Nakamura, T. Kimura, Y. Nakamichi, and H. Nakajima, Physica (Amsterdam) B (to be published).

12J. Pomeranchuk, JETP 11, 226 (1941).

${ }^{13}$ I. Dzyaloshinskii, A. Polyakov, and P. Wiegmann, to be published.

${ }^{14}$ P. W. Anderson, G. Baskaran, Z. Zou, and T. Hsu, Phys. Rev. Lett. 26, 2790 (1987); G. Baskaran, Z. Zou, and P. W. Anderson, Solid State Commun. 63, 973 (1987).

${ }^{15}$ A. E. Ruckenstein, P. J. Hirschfield, and J. Appel, Phys. Rev. B 36, 857 (1987)

${ }^{16}$ Y. Isawa, S. Maekawa, and H. Ebisawa, Physica (Amsterdam) B (to be published).

${ }^{17}$ Y. Suzumura, Y. Hasegawa, and H. Fukuyama, to be published.

${ }^{18} \mathrm{P}$. W. Anderson, in Proceedings of the Enrico Fermi International Summer School of Physics on Frontiers and Borderlines in Many Particle Physics, Varenna, Italy, 1987, edited by
R. A. Broglia and J.R. Schrieffer (North-Holland, Amsterdam, to be published).

${ }^{14} \mathrm{P}$. Gutsmiedl, K. Andres, B. Amberger, and H. Rietschel, Jpn. J. Appl. Phys. 26, 1117 (1987).

${ }^{20}$ A. Junod et al., Jpn. J. Appl. Phys. 26, 1119 (1987).

${ }^{21}$ C. Ayache, B. Barbara, B. Bonjour, P. Burlet, R. Calemczuk, M. Couoch, M. J. G. M. Jugens, J. Y. Henry, and J. Rossat-Mignot, to be published.

${ }^{22}$ M. E. Reeves, T. A. Friedmann, and D. M. Ginsberg, Phys. Rev. B 35, 7207 (1987); B. D. Dunlap, M. V. Nevitt, M. Slaskiu, T. E. Klippert, Z. Sungaila, A. G. McKale, D. W. Capone, R. B. Poeppel, and B. K. Flandermeyer, Phys. Rev. B 35, 7210 (1987); L. E. Wenger, J. T. Chen, G. W. Hunter, and E. M. Logothetis, Phys. Rev. B 35, 7213 (1987); S. J. Collecott, G. K. White, S. X. Dou, and R. K. Williams, Phys. Rev. B 36, 5684 (1987).

${ }^{23}$ F. J. Walker and A. C. Anderson, Phys. Rev. B 29, 5881 (1984).

${ }^{24}$ R. C. Zeller and R. O. Pole, Phys. Rev. B 4, 2029 (1971); D. A. Ackerman, D. Moy, R. C. Potter, and A. C. Anderson, Phys. Rev. B 23, 3886 (1981); W. N. Lawless, T. Nakamura, M. Takashige, and S. L. Swartx, J. Phys. Soc. Jpn. 52, 207 (1983).

${ }^{25}$ Y. Maeno, Y. Aoki, H. Kaminura, J. Sakurai, and T. Fujita, Jpn. J. Appl. Phys. 26, L402 (1987).

${ }^{26} \mathrm{~K}$. Kumagai, H. Aoki, I. Watanabe, and Y. Nakamura, to be published.

${ }^{27}$ L. C. Smedskjaer, J. L. Routbort, B. K. Flandermeyer, S. J. Rothman, D. G. Legnimi, and J. E. Baker, Phys. Rev. B 36, 3903 (1987).

${ }^{28}$ J. M. Tranquada, D. E. Cox, W. Kunnmann, H. Moudden, G. Shirane, M. Suenaga, P. Zolliker, D. Vaknin, S. K. Sinha, M. S. Alvarez, A. J. Jacobson, and D. C. Johnston, to be published. 